\title{
Careful Development Can Enhance LED Luminaire for Office Lighting
}

\author{
Abdelhalim Zekry \\ Ain shams university, Cairo, \\ Egypt
}

\author{
Sarah A. Elariane \\ Housing and Building National \\ Research Center, Cairo, Egypt
}

\author{
Hoda Elsharkawy \\ Ain shams university, Cairo, \\ Egypt
}

\begin{abstract}
The goal of the study in this paper is to develop a new LED luminaire consisting of rectangular arrangement of power LEDs for office lighting. The new luminaire has improved cooling arrangement to reduce its operating temperature. The luminaire is specified, designed, constructed and tested according to custom made measuring methods and setups. The same measurements have been carried out on a commercial luminaire having an equal output light for sake of comparison. The designed LED luminaire is much lighter than the commercial. Here it is proved that with careful design one can further improve the construction of the LED luminaire.
\end{abstract}

\section{General Terms}

Performance of solid state lighting luminaire

\section{Keywords}

Board, Driver, Heat sink, High power LEDs, LED lighting, LED luminaire, LED, PCB

\section{INTRODUCTION}

There are always advancements in the field of lightening where it represents approximately about $30 \%$ of electricity consumption in most types of buildings [1]. The incandescent lamps represent the oldest type followed by the gas filled lamps such as fluorescent lamps and recently the LED lamps appear [2].The LED lamps are based on the light emitting diodes and have superior optoelectronic performance. In the sense, they have high conversion efficiency and long lifetime. Obviously, there are many researches trying to design LED luminaires. They began to replace the old lamps such as incandescent lamps that emit light by rely on heated filament as well as fluorescent lamps that produce light by using gas discharge to excite phosphors, and HID lamps that use an electric arc discharge. This paper shows the design and development of LED luminaries based on advanced light emitting diodes [3]. Over past years LED Lighting technology has taken important role in science and technology development because of their major benefits for the environment and end-consumers of illumination products which do not emit much ultraviolet or infrared radiation. LED lighting technology is green lighting technology because of its clear environmental benefits and safety of normal use which has immense advantages. The major advantage of LED lighting technology is the energy conversion efficiency which is not affected by shape and size unlike fluorescent light lamps; LEDs lighting have more lumens per watt emitted than incandescent light lamps. As the LEDs produce light without using color filters, unlike traditional lighting, they have low initial cost. The LED lighting is very easy to dim by pulse modulation or by lowering its forward electric current since the on-off time is in the order of microsecond. There are some additional factors that favor the LED lighting as their small size for assembling in PCB; the cycling which is ideally used for repeating on-off cycles unlike fluorescent lamps and HID lamps which have a long restarting time. LEDs radiate cool light with very little heat in the form of IR unlike most traditional light sources where IR causes the damage to sensitive objects. The LEDs are characterized by slow failure as the LEDs lighting dim over the time unlike incandescent lamps of sudden failure. The lifetime [4] of the LEDs are long ranging between 35,000 hours to 50,000 hours unlike fluorescent lamps that have about 10,000 hours to 15,000 hours lifetime and the incandescent lamps of 1,000 to 2,000 hours lifetime. The possibility for focusing LED light is by its solid package unlike incandescent and fluorescent lamps where they use an external reflector to collect and direct light. Finally the LED lamps are shock resistant unlike incandescent and fluorescent lamps [5].

HOWEVER, the commercial LED luminaires still need further development to increase their conversion efficiency. To make them much green, and to render them more cost affordable against the conventional lamps.

In this paper it is shown how to further develop LED luminaires to make them operate at appreciably lower temperature and hence increase their lifetime.

In order to achieve the objective of the paper the following tasks are implemented: a conventional lamp for office lighting is selected. Then a commercial LED luminaire with the required specifications is chosen from the international LED lighting sources as a guide for designing a new one. The characteristics of the commercial LED luminaire are experimentally investigated. Then methods are devised to produce an enhanced LED luminaire with less operating temperature. The produced luminaire is tested and compared to the commercial one. The following sections in the paper will present in detail the above tasks.

\section{METHODOLOGY}

A fluorescent tube, TL38W/D, with $40 \mathrm{~W}$ and $28501 \mathrm{~m}$ is selected as a conventional lamp for office lighting [6]. An enhanced, designed, LED luminaire using high power LEDs [7] is used to replace this fluorescent lamp as producing the same lumens, but is more energy efficient.

This lamp is to be substituted with a LED luminaire outputting the same light quantity measured in lumens. The LED luminaire is normally consisted of power LEDS with every LED producing about 90 to 100 lumens. So, to produce 2850 lumens as of the fluorescent, the LED luminaire is built from 30 such power LEDs approximately. Therefore, 30 high power LEDs produce 2700 lumens which is very near to the intended value as illustrated in Figure 1. 

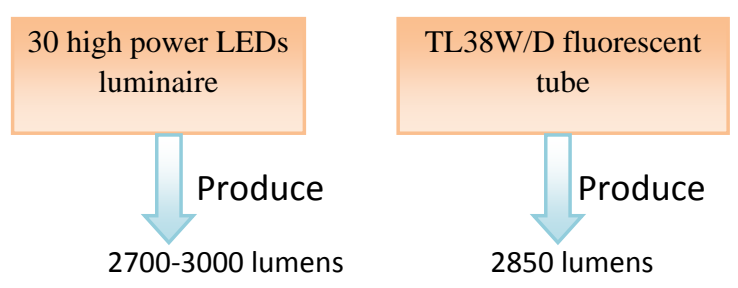

Fig 1: lumens from fluorescent lamp and LED luminaire

Accordingly, TL38W/D fluorescent tube can be replaced by a LED luminaire with 30 high power LEDs for obtaining more or less the same lumens. The first step in producing the proposed enhanced luminaire is to choose a commercial LED luminaire and investigate it. This will be done in the next section.

\section{THE CHARACTERISTICS OF THE COMMERCIAL LED LUMINAIRE:}

In this section it is introduced the selected commercial luminaire and investigate it to work out its performance characteristics and get out its advantages and disadvantages. This is done to serve as a guide for designing and testing an enhanced one. Any LED luminaire consists of four parts, namely, the LEDs assembled on a board, a heat sink, an array lens to diffuse the light, and a power supply. Accordingly, it is observed that any LED luminaire has the following specifications parameters:

a) The board type connecting the LEDs.

b) The external shape of the board and its dimension.

c) The number of LEDs used in the luminaire. d) The distance between LEDs.

e) The circuit connection of the LEDs

f) The type and the shape of the heat sink.

g) The type of the luminaire lens used to diffuse the light.

h) The power supply that is used with it.

i) The output light or the lumens produced.

\subsection{Physical characteristics of the chosen LUMINAIRE}

The board type supporting the high power LEDs, the external shape of the board and its dimensions, the number of the high power LEDs used in this design, the distance between them, and the circuit connection of the LEDs (parallel or series connection) are illustrated in Figure 2.

The heat sink is made of aluminum with the shape illustrated in Figure 3.

The array of lens for the commercial LED luminaire is illustrated in Figure 4.

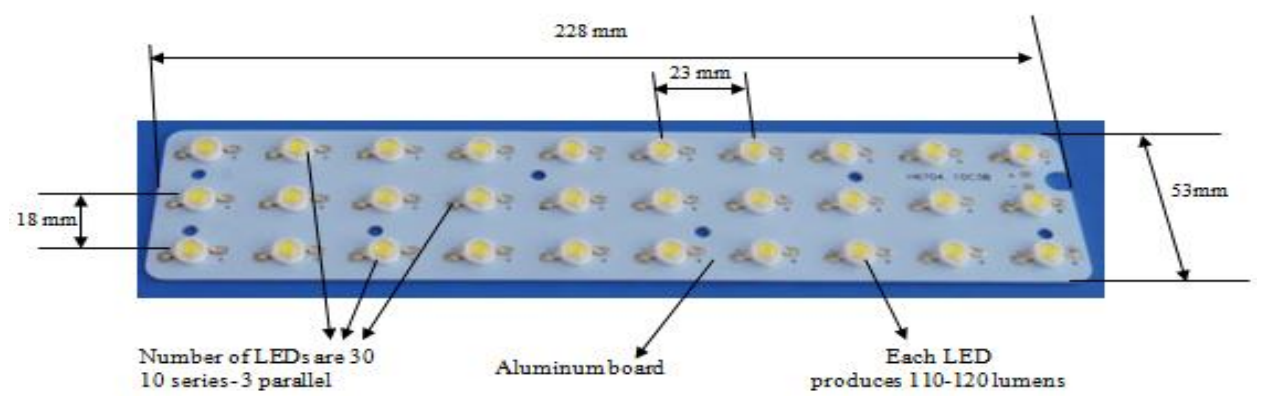

Fig 2: The board of the of the commercial LED luminaire

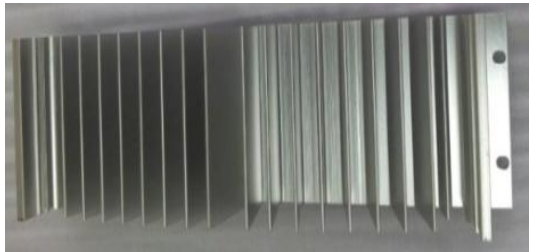

Fig 3: heat sink of the commercial LED luminaire

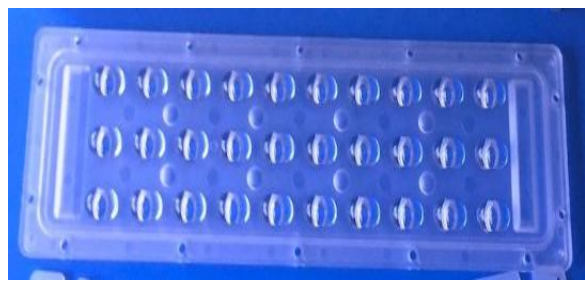

Fig 4: Array lens of the commercial LED luminaire
The driver with waterproof aluminum case of the commercial LED luminaire is shown in Figure 5. The driver is a DC power supply with AC input voltage of $110-220 \mathrm{~V}$, a DC output voltage of $30-36 \mathrm{~V}$, and dc current of $960 \mathrm{~mA}$.

In the following sections the properties of this commercial luminaires will be experimentally investigated in a comprehensive manner. Therefore many experimental set ups are devised to perform measurements. 


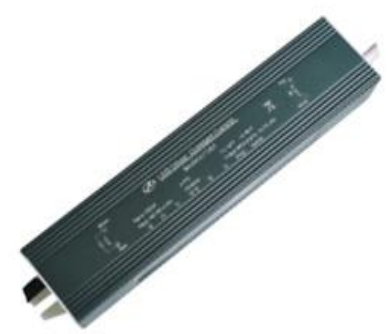

Fig 5: photo of driver of commercial LED luminaire

\subsection{Electrical Characteristics}

The first characteristic to be measured is the I-V characteristics of the commercial LED luminaire.

$\mathrm{I}-\mathrm{V}$ measuring circuit is shown in Figure 6 where the luminaire is fed by its power supply through variable AC transformer, VARIAC, connected to the mains utility AC supply. The DC voltage across the luminaire is measured by a voltmeter and the current is measured by an ammeter. The set up is illustrated by the photograph shown in Figure 7. The VARIAC is used to vary the AC input to the luminaire and hence a whole I-V curve can be measured.

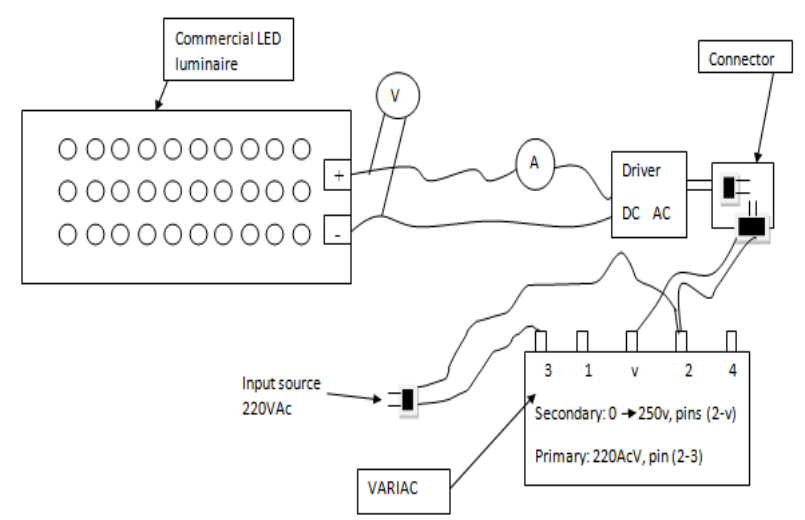

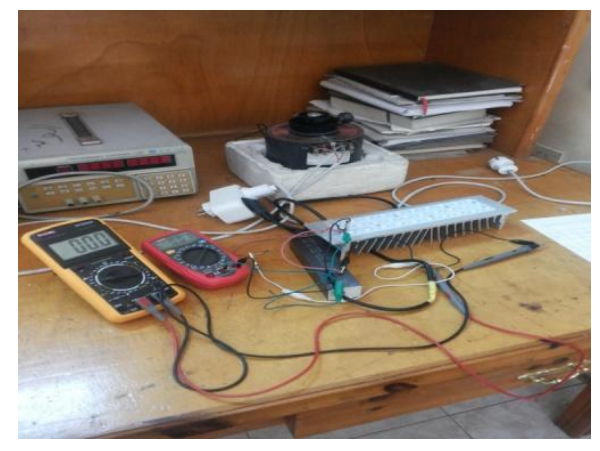

Fig 7: Photo of the I-V measuring set up of the LED luminaire

The measured I-V characteristic is plotted in Figure 8 on linear scale. It is clear from the figure that luminaire withdraws a current of $950 \mathrm{~mA}$ at a luminaire voltage of 32.5 $\mathrm{V}$. This is in agreement with the given data sheet of the device. Accordingly, the power consumption of the device is $30.8 \mathrm{~W}$. For sake of completeness and fair comparison, the I$\mathrm{V}$ characteristics of one LED building the commercial luminaire is measured in the same manner except the LED is fed from a suitable variable DC power supply. So, the VARIAC and the fixed DC power supply are substituted by a DC power supply. Such measurement is required also for modeling and simulation purposes. Having such characteristics, the equivalent circuit parameters of the LED can be extracted.

Fig 6: Measuring set up of the I-V characteristics of the commercial LED luminaire

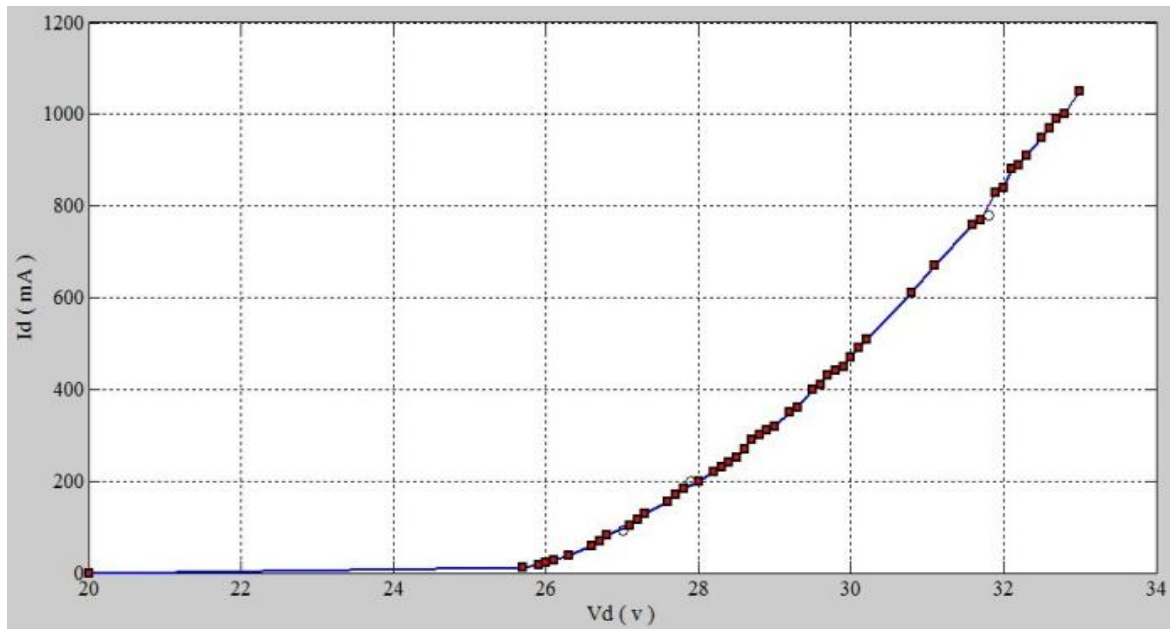

Fig 8: The measured I-V characteristics of the commercial LED luminaire 


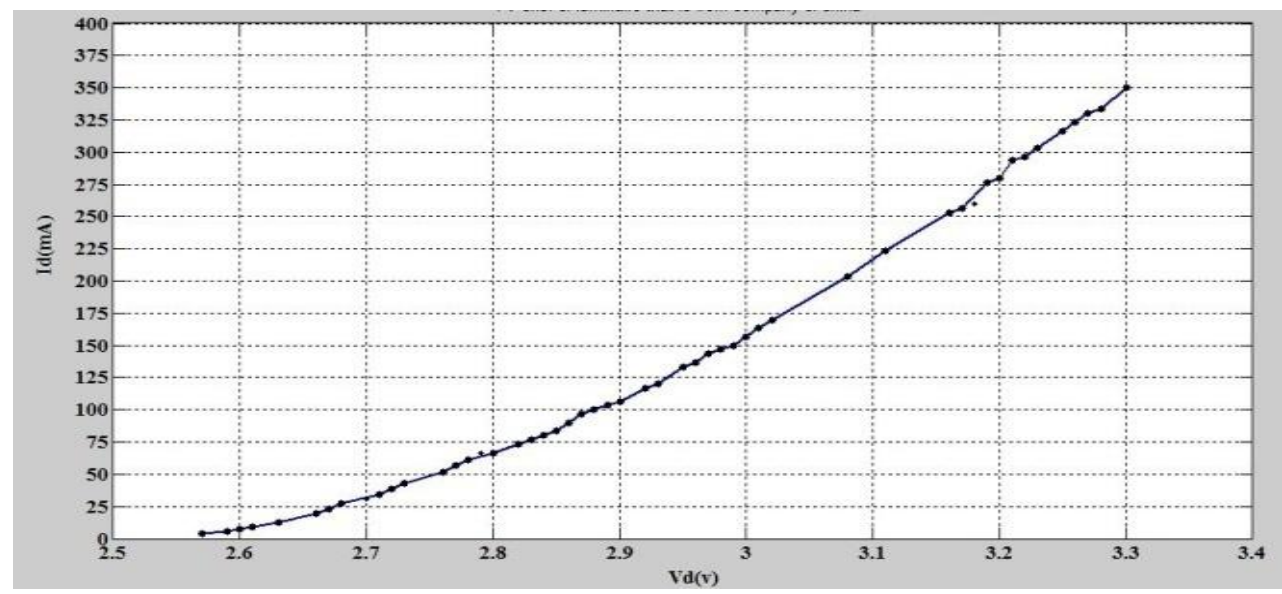

Fig 9: I-V characteristics curve of high power LEDs used in the commercial LED luminaire

The results of the measurement are depicted in Figure 9. The high power LED used in the commercial LED luminaire drops a $3.25 \mathrm{VDC}$, at a current of $316.7 \mathrm{~mA}$ with a power consumption of $1.09 \mathrm{~W}$.

One important notice here is that the diode withdraws only an appreciable current first at a cut in voltage of $2.5 \mathrm{~V}$ and the curve is highly nonlinear with an appreciable differential resistance. It does not look as a rectifier diode in the sense that its current increases by large amount for a very incremental change of the voltage. This is a very important result concerning the driver characteristics of the power LED. It must not be fed by a constant current source as in the practice till now. The power LEDs can be driven by a constant voltage source. This makes the driver more power efficient. This is because the supplies are normally voltage sources and one converts them to current sources.

\subsection{Optoelectronic Characteristics of the commercial Luminaire}

The optoelectronic properties of the luminaire and its building diodes are measured by an innovative method where the photo flux emanating from the part of the luminaire is received by a solar cell and its photocurrent Iph is measured and considered as an indicator for the emitted lumens of the luminaire. The idea of this measurement has been taken from a previous paper [8]. The electrical quantify of interest is the luminaire current IL. The proposed experiment aim is to measure the relationship between the corresponding photocurrent and average current to one LED, 15 LEDs, and the total commercial LED luminaire. The solar cell is used to detect the incident photon flux produced in each case. The one LED, 15 LEDs, and the total commercial LED luminaire are inserted in a dark box in the same axis to a solar cell to measure the corresponding photocurrent for the three cases respectively. The dark box is used to guarantee that all light produced from the three cases is incident to the solar cell. The set up to carry out this test is depicted in Figure 10 where it contains the same components as in the previous set up in addition to the large area solar cell acting as a photodetector.

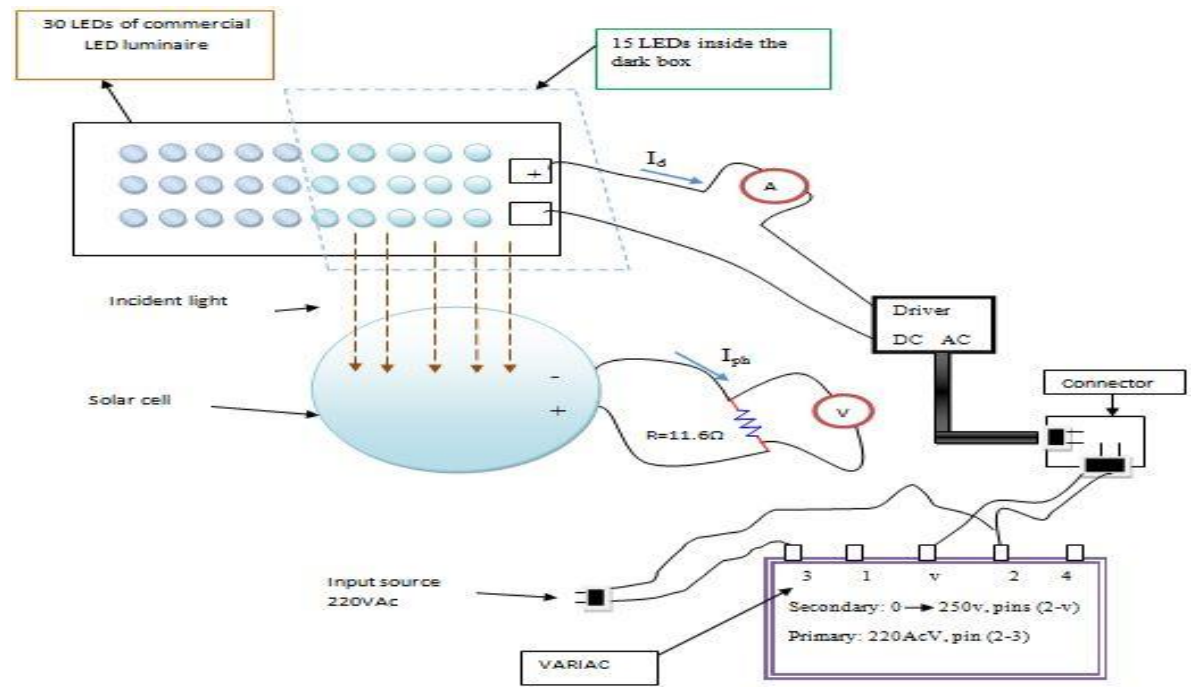

Fig 10: circuit diagram for measuring the corresponding photocurrent against the average LED current for 15 LEDs

The photocurrent is measured by shunting the solar cell with a current sensing resistance and the voltage across it, is measured by a dc voltmeter and the current is calculated.
The results of the measurement are shown in Figure 11 for the three cases, the total photon flux from the luminaire as a function of the average LED current say case 1, 15 LEDs as a 
function of their average diode current, case2, and finally case 3 for one LED photon flux against its biasing current.

We see that as the LED biasing increases the photocurrent increases in a nonlinear manner. In the range of higher LED current, the slope of the curve decreases indicating the reduction of the emission efficiency which can be indicated by the change of the photocurrent by a given change in the bias current of the LED. This is a well known phenomenon for LEDs. It is also apparent from the figure that the emitted photon flux is multiplied by the number of active diodes. This proves that diodes are nearly homogeneous concerning their optoelectronic properties.

\subsection{Thermal Characteristics}

The thermal properties of the luminaire are very important as they give an indication about the thermal losses and the rise in temperature because of feeding the LEDs with electrical power.

The supplied electrical energy is converted partly to light and partly to heat. The heat energy leads to a temperature rise in the LED luminaire. The temperature of the LED luminaire [9] is measured to know the energy loss as heat. The LED luminaires have to be mounted on a heat sink to remove unwanted heat because over heating is life shortening and even may be destructive. So, it is required to reduce the operating temperature of the luminaire.

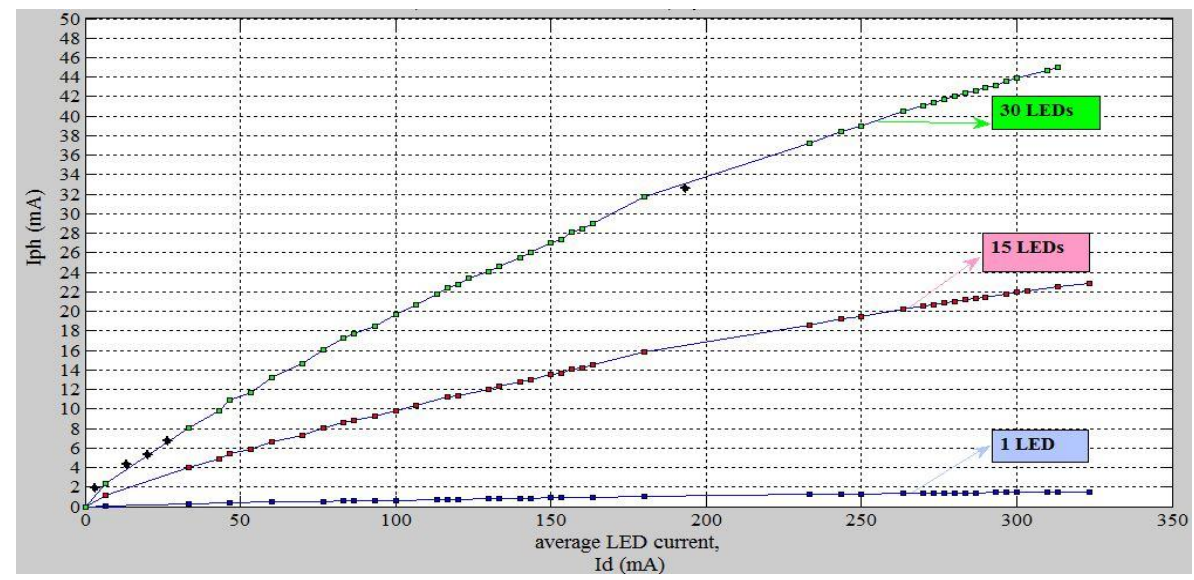

Fig 11: Measured relationship between corresponding photocurrent and average current for one LED, 15 LEDs, and the total 30 LEDs of the commercial LED luminaire

To evaluate the heat sink effectiveness of the commercial luminaire, it is operated under nominal condition of $950 \mathrm{~mA}$. Then the temperature for the commercial LED luminaire is measured by thermistors after one hour from switching on the power supply. The maximum temperature and the average temperature for the LED luminaire as illustrated in Figure 12 are $33.3^{\circ} \mathrm{C}$ and $30.9^{\circ} \mathrm{C}$ respectively, knowing that the ambient temperature in the laboratory was $12.6{ }^{\circ} \mathrm{C}$. Thus, this temperature rise may be relatively high and in hot days of about 30degree centigrade the temperature of the case may reach about fifty degrees which may have detracting effect on the luminaire. So, better luminaires are required. In order to compare the cooling of this luminaire with the developed one its temperature is measured at an operating current of $180 \mathrm{~mA}$ and it is found that its temperature arrived at 19.5 degree centigrade.

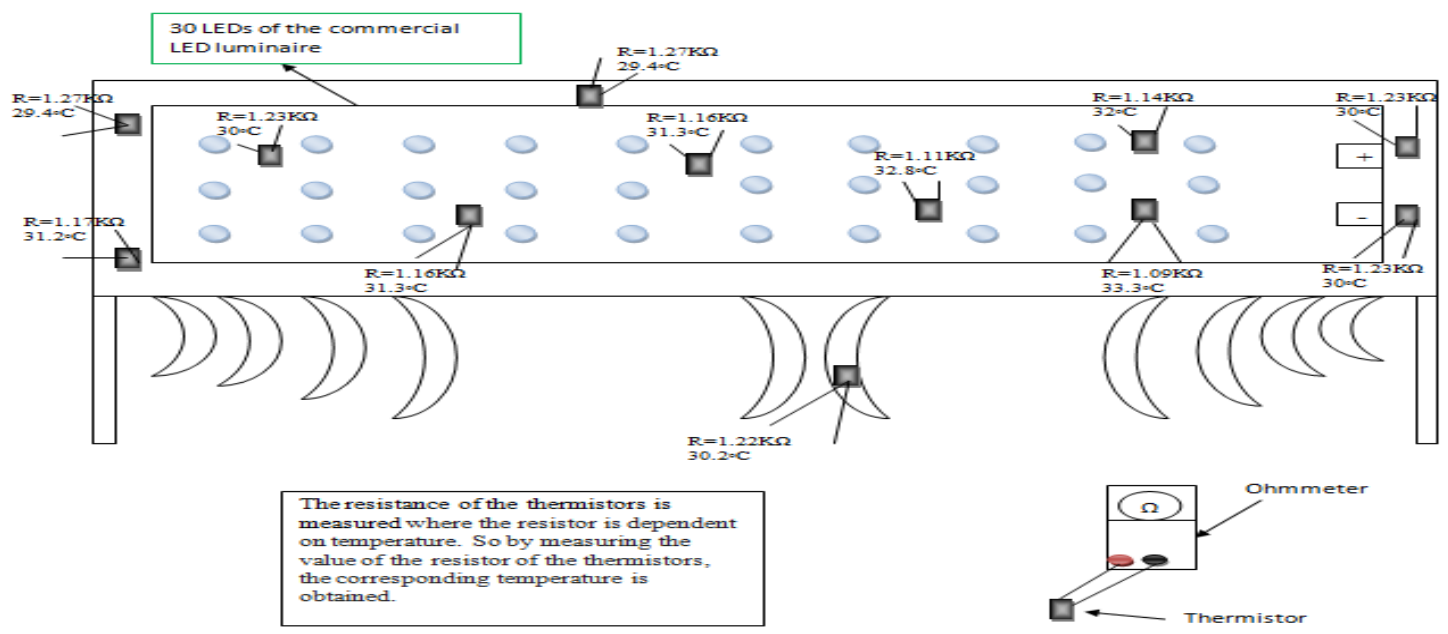

Fig 12: The locations of thermistors in measuring temperature for LED luminaire

\subsection{The SPICE Parameters of the Led}

In order to simulate the performance of the luminaires using the circuit simulator SPICE, the spice parameters of the power LEDs has to be extracted forming the luminaire. This is very useful for simulating the whole luminaire even with heat sink. 
The parameters of the LED diode resemble any diode parameters which are based on the well known diode equation:

$$
I_{L}=I_{d}=I_{S}\left(e^{\frac{V d-I d R s}{\eta V t}}-1\right)+V / R_{\text {sh }} \text {, with the parameters: }
$$

$I_{S}$ : the reverse saturation current, i.e., the current value when $\mathrm{V}_{\mathrm{d}}=0$

$\mathrm{I}_{\mathrm{d}}$ : current flowing through the LED

$\mathrm{V}_{\mathrm{d}}$ : applied voltage across the terminals of the LED

$\eta$ : emission coefficient

q: absolute value of electron charge

$\mathrm{K}$ : Boltzmann's constant

T: absolute temperature in Kelvin $(\mathrm{K})$

$\mathrm{V}_{\mathrm{t}}=25.8 \mathrm{mV}$ at $\mathrm{T}=300 \mathrm{~K}$

$\mathrm{R}_{\mathrm{s}}$ : the series resistance of the diode

$\mathrm{R}_{\mathrm{sh}}$ : the shunt resistance of the diode

The shunt resistance dominates the I-V characteristics at very small voltages near the zero voltage bias. It is determined first and found to be $R_{\text {sh }}$ equals to $0.594 \mathrm{~K} \Omega$. Then its current is subtracted from measured diode current to eliminate it effect from the I-V curve.

The exponential term is much greater than 1 for $\mathrm{V}>3 \mathrm{Vt}$ and at low currents the Id Rs drop is negligible, therefore, the diode equation reduces to:
$\mathrm{I}_{\mathrm{d}} \approx \mathrm{I}_{\mathrm{S}} \mathrm{e}^{\frac{\mathrm{Vd}}{\eta \mathrm{Vt}}}$

Plotting the measured diode characteristics on a semi logarithmic scale, then the saturation current $I_{s}$ and the ideality factor $n$ can be obtained, $\eta$, by curve fitting with the relation:

$\ln \left(\frac{\mathrm{Id}}{\mathrm{Is}}\right)=\frac{\mathrm{Vd}}{\eta \mathrm{Vt}}$

It has been found that $I_{s}=2.096213 * 10-21 \mathrm{~A}, \mathrm{n}=2.43205$.

The series resistance Rs is determined from the extrapolation of the exponential relation at higher current and then considering that the measured voltage is deviated from that of the exponential by the voltage drop $I_{d} R_{s}$. From this deviation at certain current we get $R_{s}$. It is found that $R_{s}=1.4 \Omega$.

\subsection{Verification of the measurement result by Software Simulation}

To implement and to verify the characteristics of the commercial LED luminaire, the TopSpice [10] program is used.

The SPICE parameters of the LEDs in the commercial luminaire that are previously extracted are inserted in TopSpice code of the netlist file.

The measured I-V characteristics and the simulated I-V characteristics are compared for individual LED and for the whole LED luminaire as shown in Figure 13 and Figure 14, respectively.

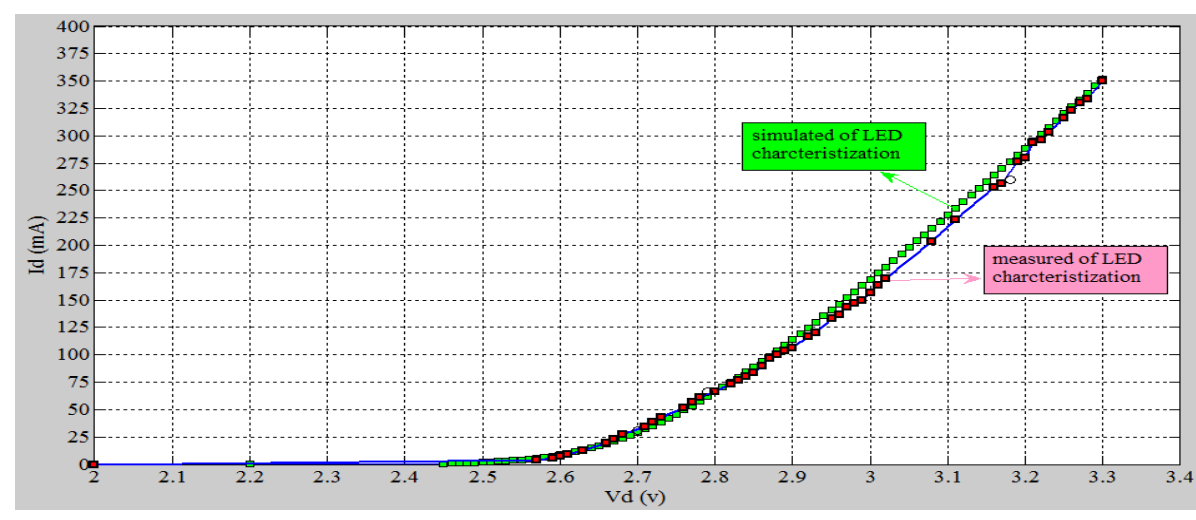

Fig 13: The measured and simulated I-V characteristics of individual LED used in the commercial luminaire

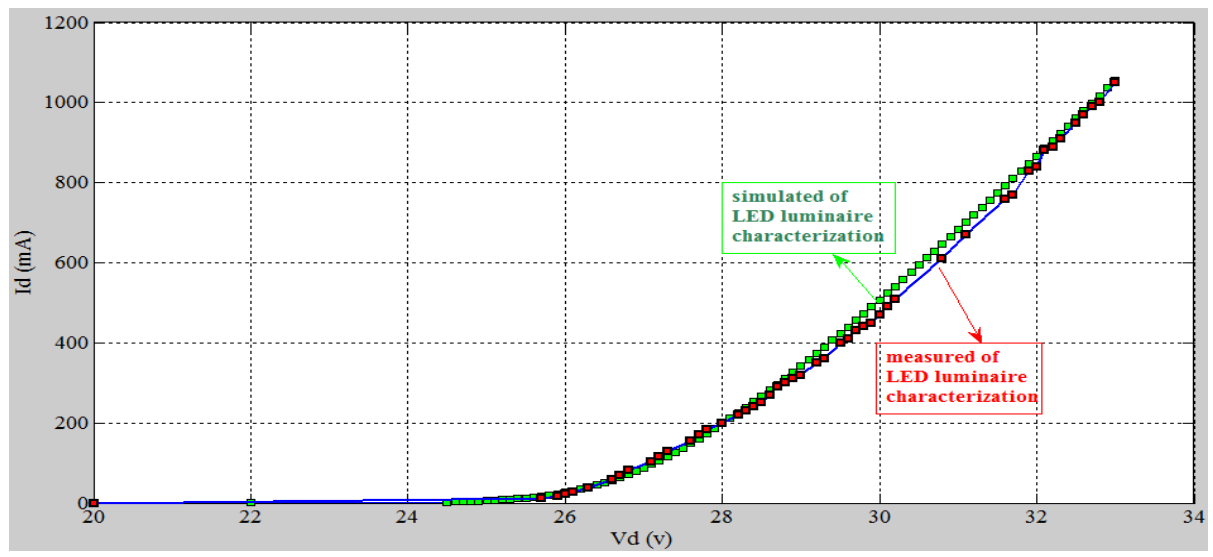

Fig 14: The measured and simulated I-V characteristics for the commercial LED luminaire 
It is clear from the figure that there is satisfactory agreement between the measured and simulated results for the single LED and for the luminaire. These points out that, this model fairly describes the I-V characteristics of the diode. Then such model can be used to design the power supply for any other luminaire with different number of LEDs.

\section{THE CHARACTERISTICS OF THE ENHANCED LED LUMINAIRE}

\subsection{Physical characteristics}

It is wanted to produce a LED luminaire which has better cooling than the commercial one to operate it at lower temperature of LEDs and increase the lamp life time.
The board type connecting the LEDs, the external shape of the board and its dimensions, the number of LEDs used in this design, the distance between LEDs, and the circuit connection of the LEDs [11] are illustrated in Figure 15. The LEDs are assembled on double sided printed circuit board with the same dimensional array of LEDs as in the commercial luminaire.

The printed circuit board is coated with transparent insulating layer and then it is covered with Aluminum foil to aid in dispatching the heat from the LEDs to the metal heat sink and thereby affecting better cooling of the luminaire. These are major modifications on the original construction of the luminaire.

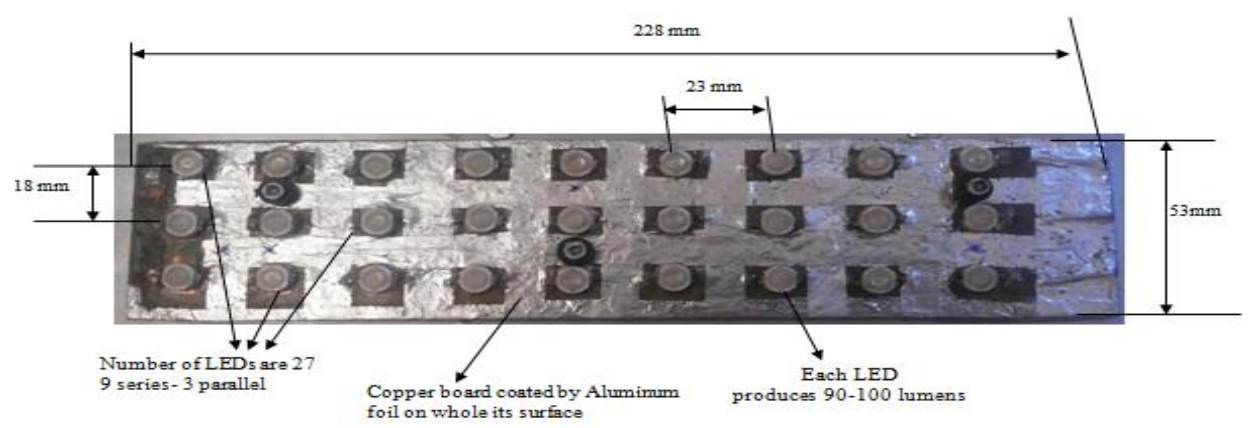

Fig 15: The array of LEDs on the PCB for proposed LED luminaire

The type of heat sink of the designed LED luminaire is aluminum and its shape is simple U- shaped as illustrated in Figure 16.

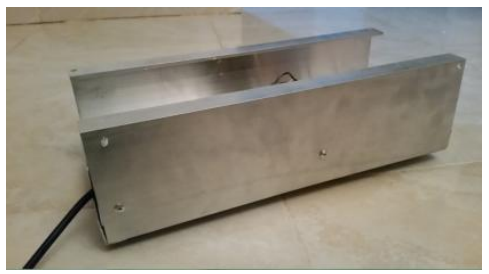

Fig 16: heat sink of designed LED luminaire

The array of lens of the designed LED luminaire covering the LED matrix is illustrated in Figure 17.

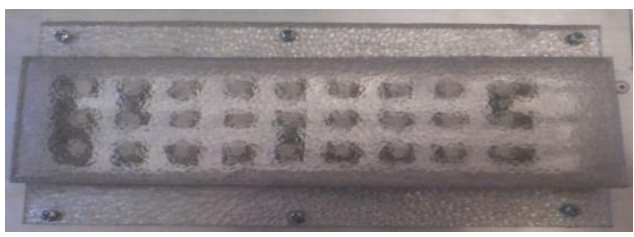

Fig 17: array of lens of designed LED luminaire

The driver of the designed LED luminaire is depicted in Figure 18. It has an input of AC $220 \mathrm{~V}$, a DC output voltage of $25.3 \mathrm{~V}$, and a DC current of $180 \mathrm{~mA}$ as required to feed the luminaire at its nominal operating conditions. It is a simple DC power supply consisting of a step down transformer, a bridge rectifier and a smoothing capacitor. The current is adjusted by a series resistance with the luminaire.

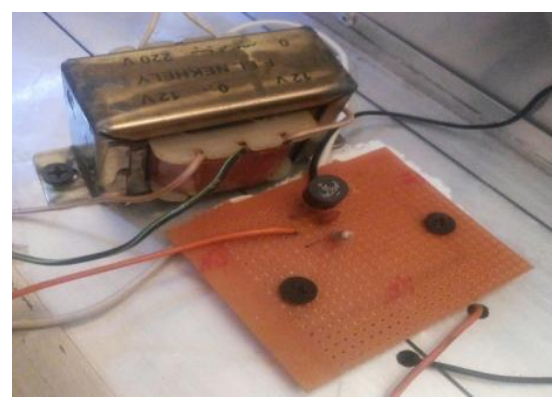

Fig 18: driver of designed LED luminaire

Following the same experimental procedures with the commercial luminaire, the proposed luminaire is fully characterized.

\subsection{Electrical Characteristics Of The Designed LED Luminaire}

$\mathrm{I}-\mathrm{V}$ characteristic of the designed LED luminaire is measured in the same way as has been done with the commercial luminaire. So, there is no need to repeat the description of the experimental work. Here the results are only displayed.

The measured I-V characteristic of the designed LED luminaire is plotted on a linear scale in Figure 19. It has a nonlinear shape characteristic of the LED luminaires. The luminaire current will be only remarkable at a luminaire voltage greater than about $22 \mathrm{~V}$. This means that the cut in voltage of the lamp will be around such value. It is noticed that the current levels of this luminaire is smaller than that of the commercial one and the operating voltage is smaller. According to the specifications of the one LED, its nominal current is $60 \mathrm{~mA}$ and yields 90 to 100 lumens for about one watt power consumption which means that it has the same light efficacy as that of the commercial LED luminaire but its maximum current is much smaller than that of the commercial LED luminaire. So, as the luminaire consists of 3 branches in 
parallel with each branch containing 9 LEDs, then the luminaire nominal operating condition is $180 \mathrm{~mA}$. From the I-V curve the corresponding voltage will be $25.3 \mathrm{~V}$. Accordingly, the total power consumption is $4.6 \mathrm{~W}$.

It is also required to measure the $\mathrm{I}-\mathrm{V}$ characteristics of individual LEDs building the module. So, two randomly LEDs are selected, their I-V characteristics are measured and the results are plotted on a linear graph as shown in Figure 20.
As expected there are some deviations between the characteristics of the two diodes, assuming the same typical characteristics. Such scattering is common for the electronic devices. Also, the shape of the I-V resembles that of the luminaire and can be obtained from that of the LED luminaire by scaling down through dividing the voltage by 9 and the current by 3. It is shown from the figure that the operating voltage deviation at the operating current of $60 \mathrm{~mA}$ is about 6 $\mathrm{mV}$.

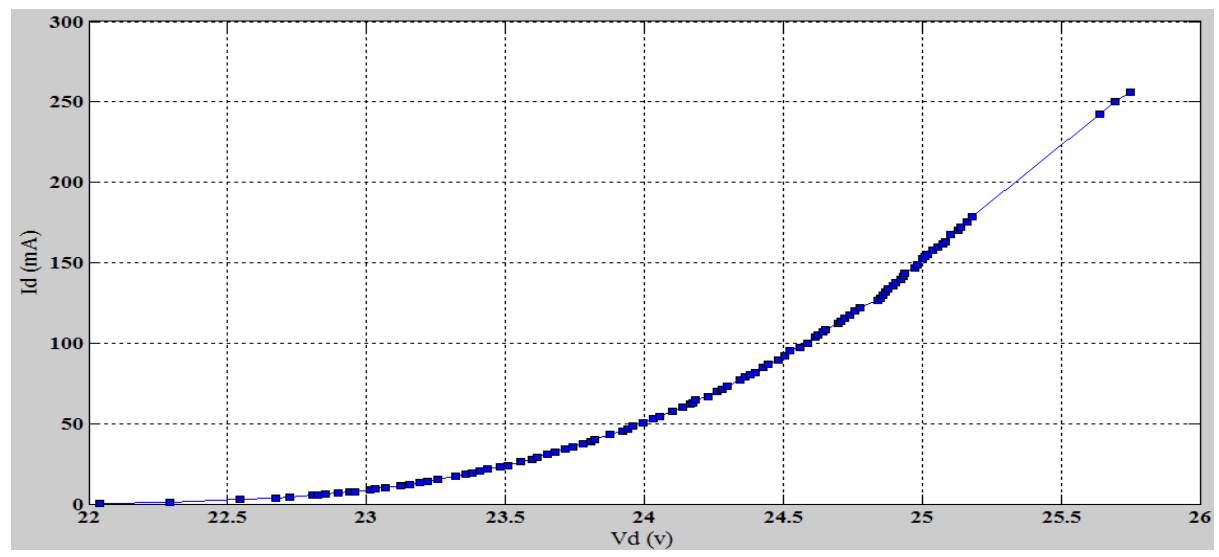

Fig 19: Measured I-V characteristics of the designed LED luminaire

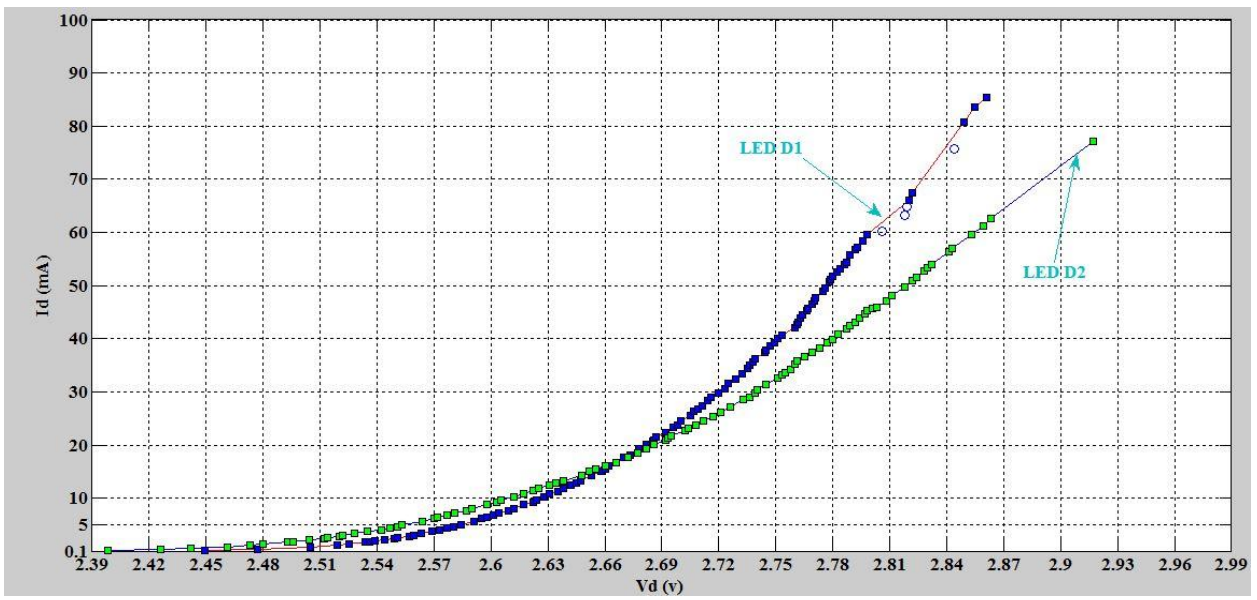

Fig 20: curves of I-V characteristics of the two high power LEDs used in designed LED luminaire

For sake of comparison, the I-V characteristics of red color LED, of miniature type, is measured and displayed in Figure 21. The shape of the curve is similar to that of power LED except that the cut in voltage is smaller than that of white LEDs used for illumination. It amounts to about $1.7 \mathrm{~V}$. Also, the operating current is smaller. 


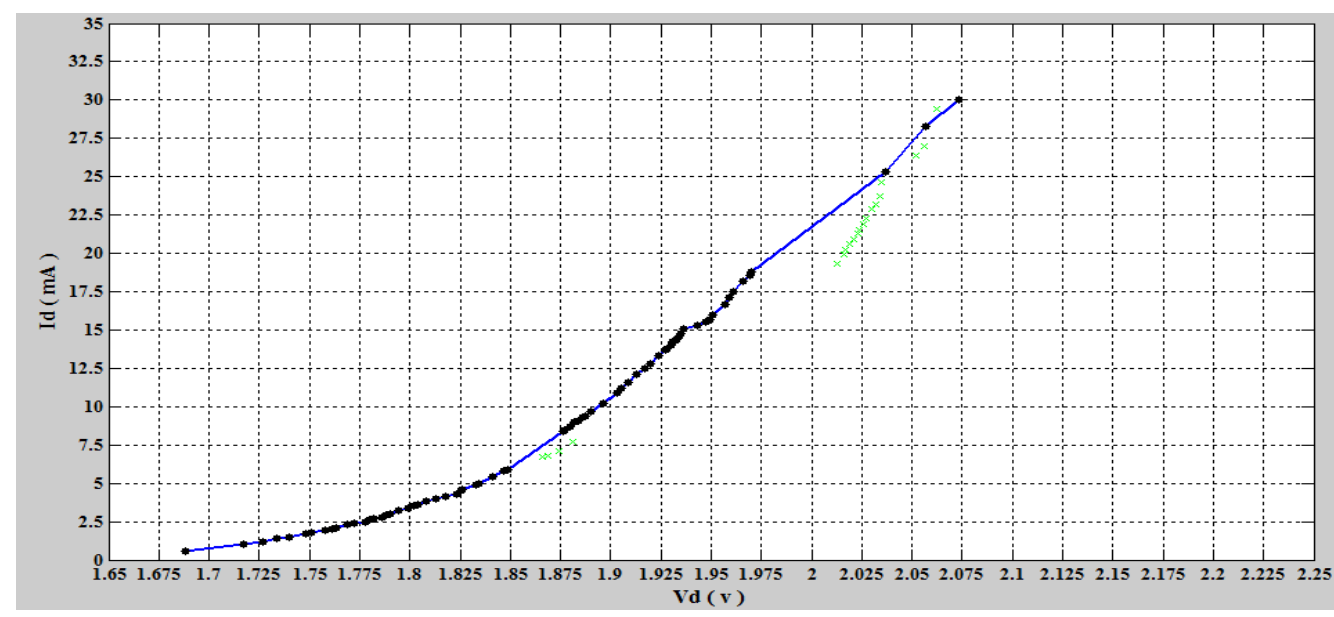

Fig 21: Measured I-V characteristics for red LED of miniature type

\subsection{Optoelectronic Characteristics Of The Designed LED Luminaire}

Now the attention is turned to optoelectronic properties of the designed LED luminaire by the same techniques used with the commercial one in the previous section.

Figure 22 shows the measured photocurrent in the solar cell photo detector as a function of average current for one, 9,18 and 27 LEDs. It is clear from the figure that the photocurrent is nonlinear with the LED diode current pointing out that the radiation efficiency of the LEDs decreases with the increase of the diode current. However, the radiation intensity is proportional to the number of active LEDs.

It is noticed that the lamp produces about the same light of the commercial luminaire when it is operated at the same current. Therefore the two lamps radiate the same power at about the same operating input power.

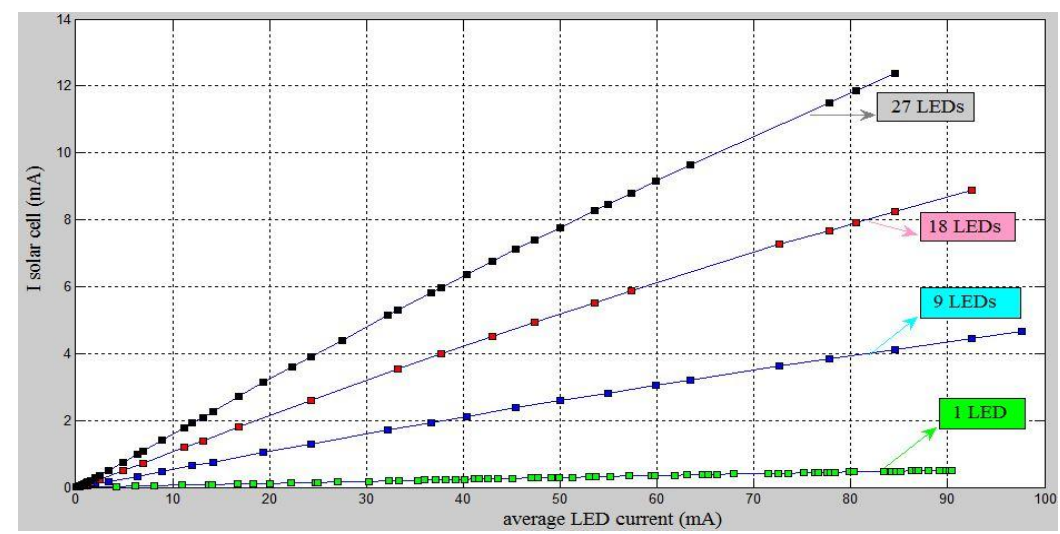

Fig 22: Measured relationship between corresponding photocurrent and average current for one LED, 9LEDs, 18 LEDs and the total 27 LEDs of the enhanced LED luminaire

\subsection{Thermal Characteristics of the Designed LED Luminaire}

The temperature of the designed LED luminaire is measured at different locations as shown in Figure 23. The luminaire temperature is measured by thermistors after one hour from switching on the power supply.
The maximum temperature and the average temperature are $38.5^{\circ} \mathrm{C}$ and $34.2{ }^{\circ} \mathrm{C}$ respectively, knowing that the ambient temperature in the laboratory is $12.6{ }^{\circ} \mathrm{C}$. The average temperature of the designed LED luminaire is relatively high compared to the ambient temperature.

Figure 24 shows the location for the thermistor to measure the time taken for the designed LED luminaire to reach the average temperature. 


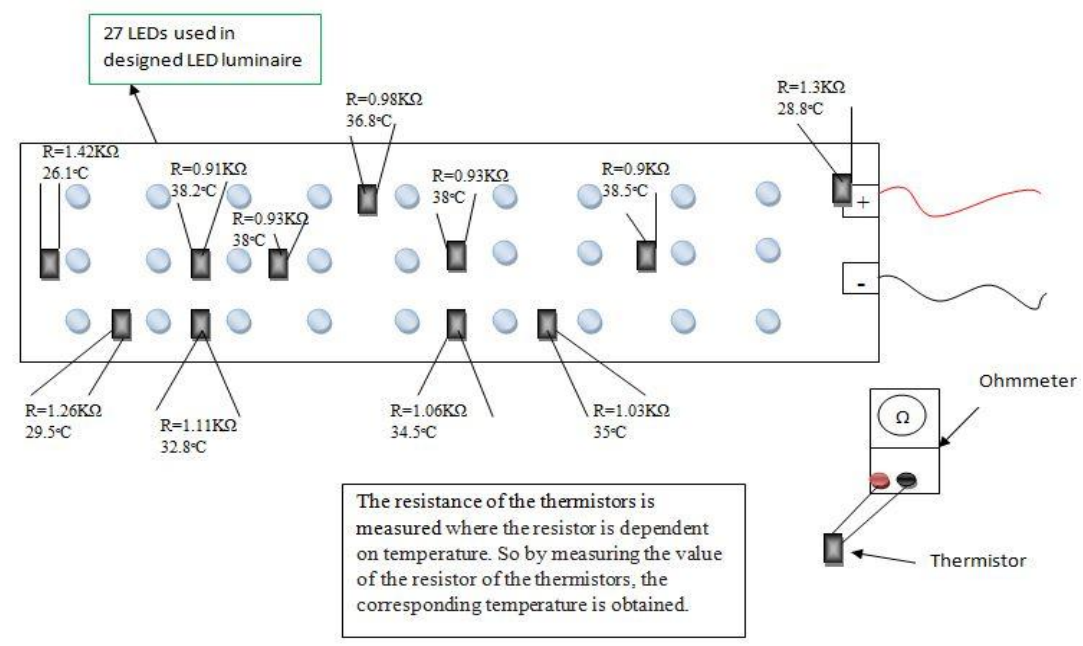

Fig 23: illustrates the locations of thermistors for measuring temperature of the designed LED luminaire before improving the cooling

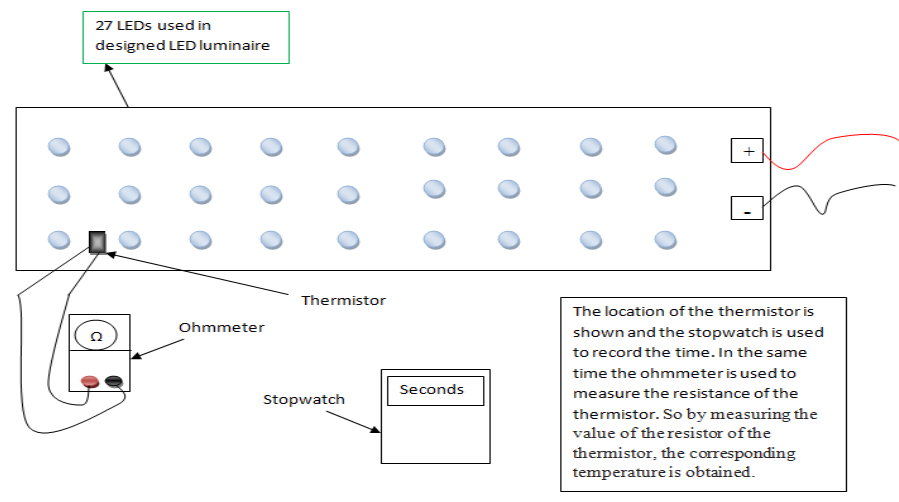

Fig 24: illustrates the location of the thermistor for measuring heating up curve of the designed LED luminaire.

An enhancement in the designed LED luminaire has been done to decrease the operating temperature. The heating up curve of the designed LED luminaire is depicted in Figure 25.

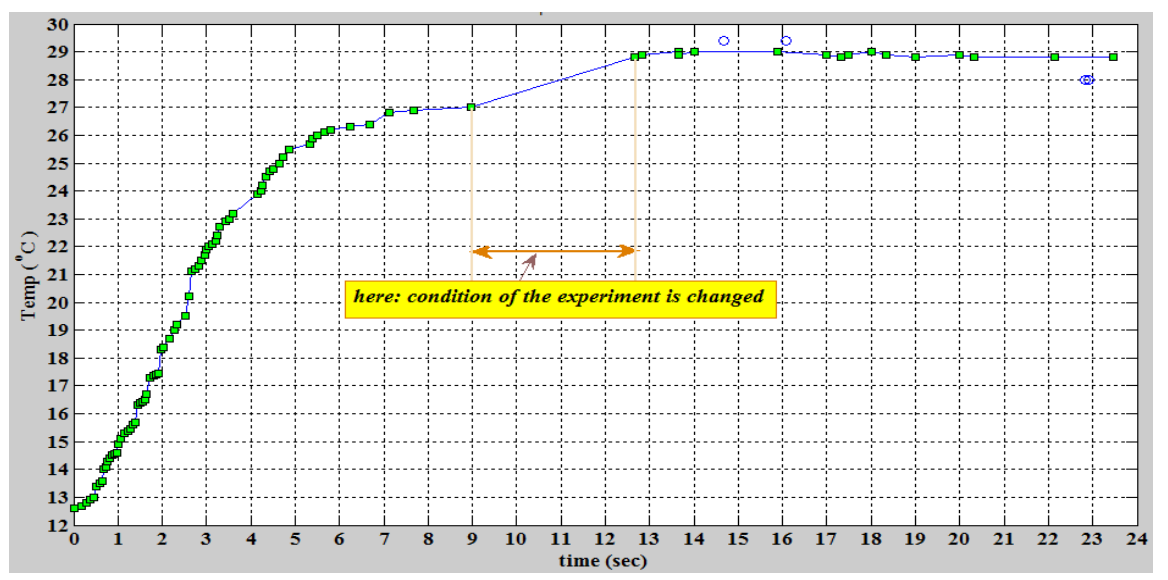

Fig 25: Measured temperature of the designed LED luminaire during heating up after switching on the power supply

It is shown from the figure that after approximately 13 seconds the temperature began to reach a steady state. It must be mentioned that as the thermistor is made more firmly contacted to test location, the second step of the temperature rise is observed. So, it can be said the luminaire reaches a steady state temperature of about 29 degree centigrade in about 8 seconds.
Thus, as this temperature is exceedingly high some provisions must be undertaken to reduce it. So, the PCB assembled with the LED array is coated with a transparent insulating film. After that an aluminum foil is adhered to the bare PCB areas to drain the heat from the LEDs conducted to the PCB surface as demonstrated in Figure 15 . The temperature of the designed LED luminaire is measured after the wrapping of the 
aluminum foil as depicted in Figure 26 following the procedures that have been done before the enhancement.

It is seen from the figure that the maximum temperature and the average temperature are $24.8^{\circ} \mathrm{C}$ and $22.5^{\circ} \mathrm{C}$, respectively. Whereas the ambient temperature in the laboratory was $13.5^{\circ} \mathrm{C}$, accordingly the enhancement achieved by adding the Aluminum foil caused the decrease of the designed LED luminaire temperature appreciably.

The decreasing in temperature is supposed to be done as the
LED luminaires are designed to operate for a long time. So, the enhancement helps the designed LED luminaire to reduce the loss of energy as heat and to operate for a long time. Then the heat sink is added for further decreasing the heat of the designed LED luminaire and is encapsulated by acrylic glass to diffuse the produced light. After the last additions, the temperature on the surface of the designed LED luminaire is measured by thermistors. It is found that the temperature of the heat sink amounts finally to $14.8^{\circ} \mathrm{C}$, which is appreciably smaller than that of the commercial LED luminaire.

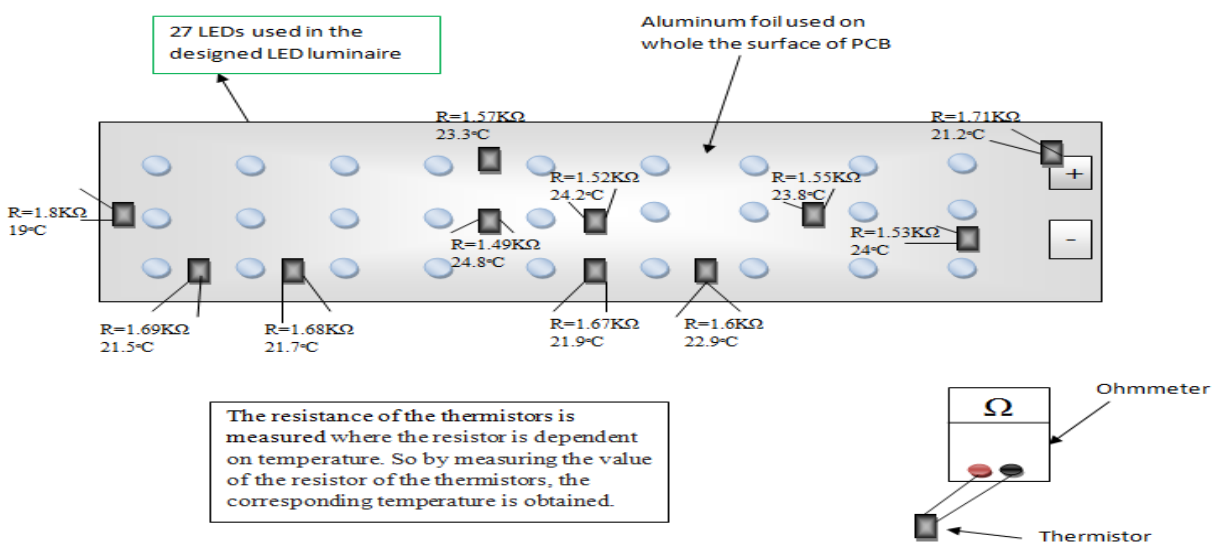

Fig 26: The places for thermistors in measuring temperature of designed LED luminaire after the enhancement

\subsection{Spice parameters of LEDs in the designed LED luminaire}

The same procedures used for the LEDs of the commercial LED luminaire are followed here to determine the SPICE parameters of the LEDs of the designed LED luminaire. By plotting the measured diode characteristics on a semi logarithmic scale, then the saturation current $I_{s}$ and the ideality factor $\mathrm{n}$ can be obtained.

Then the spice parameters are determined as listed below:

$$
\mathrm{n}=1.672
$$

$$
\text { Is }=2.8445^{*} 10-29 \mathrm{~A}
$$

$$
\mathrm{Rs}=1.4 \Omega
$$

$$
\mathrm{Rsh}=0.47 \mathrm{M} \Omega
$$

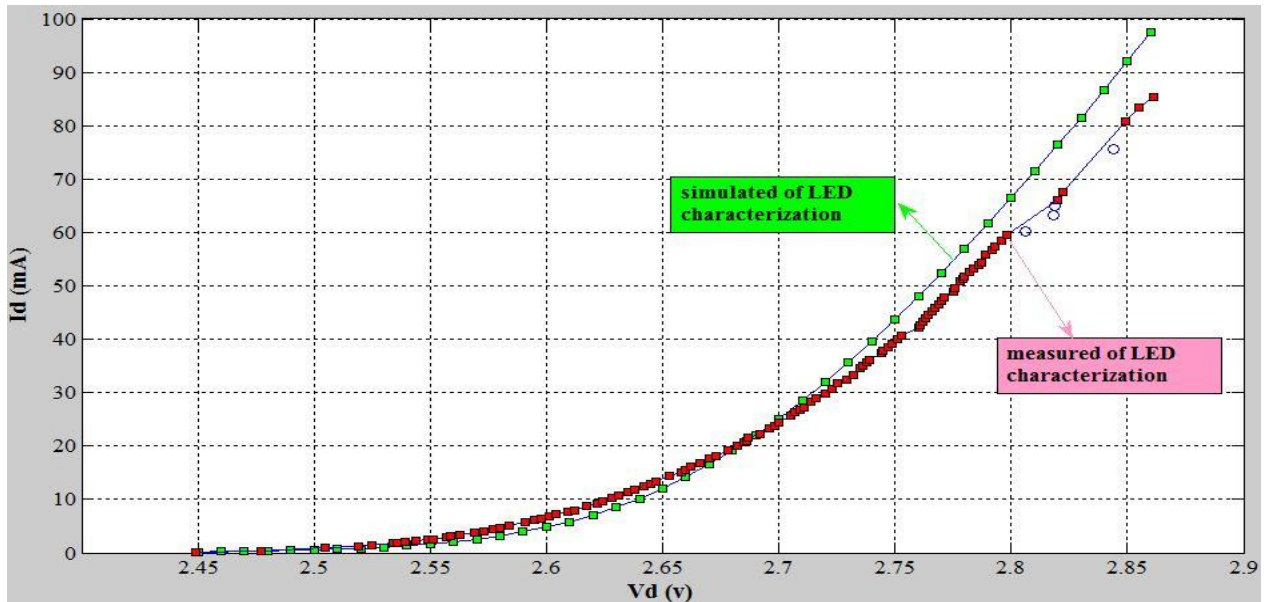

Fig 27: curve compared between measured and simulated I-V characteristics of individually LEDs used in designed LED luminaire

\subsection{Verification of the MODEL by software Simulation}

The obtained parameters of the enhanced LED luminaire are inserted in TopSpice code to plot the I-V characteristics. The measured I-V characteristics and the simulated I-V characteristics are compared for the individual LED, D1, as shown in Figure 27. In addition, the measured I-V characteristics and the simulated I-V characteristics are compared for the whole luminaire as depicted in Figure 28.

luminaire 


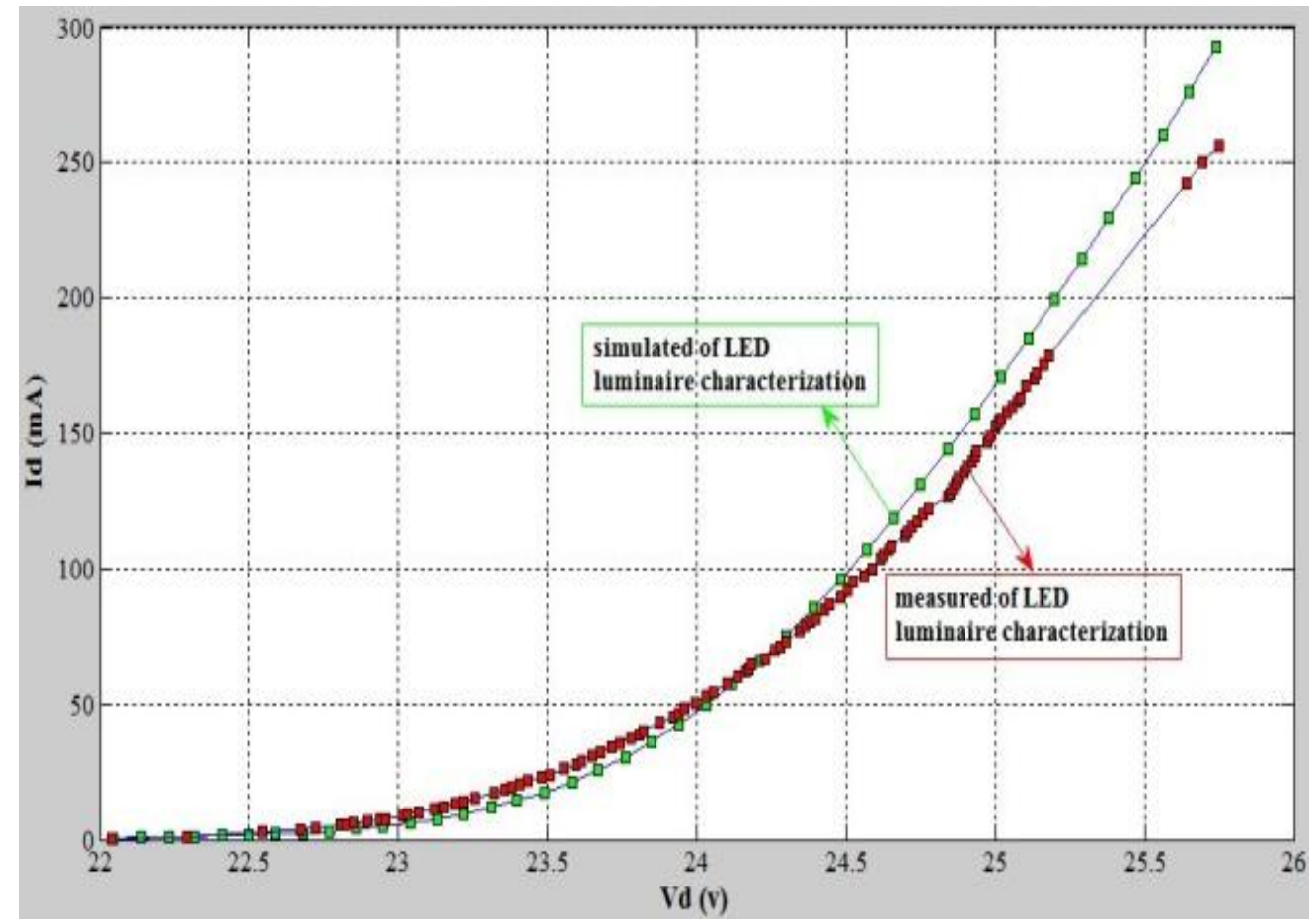

Fig 28: curve compared between measured and simulated I-V characteristics of enhanced LED luminaire

It is clear from the figures that the measured and simulated curves are in good agreement proving the correctness of the model parameters.

\section{CONCLUSIONS}

Now, the operational and performance parameters of the two investigated luminaires are listed for the same input power in Table 1 for sake of comparison. It is clear that performance parameters of the developed luminaire are better than that of the commercial one. It operates at much lower temperature. In addition the developed luminaire is lighter.

This is because of much efficient cooling heat sink. The investigations are showed that with more elaboration one can make better luminaire.

Table.1-Performance parameters of the commercial and the developed LED luminaire at the same input power.

\begin{tabular}{|c|c|c|}
\hline & $\begin{array}{c}\text { Commercial } \\
\text { LED luminaire }\end{array}$ & $\begin{array}{c}\text { Designed } \\
\text { LED } \\
\text { luminaire }\end{array}$ \\
\hline Voltage (V) & $25.3 \mathrm{~V}$ & $25.3 \mathrm{~V}$ \\
\hline Current (mA) & $180 \mathrm{~mA}$ & $180 \mathrm{~mA}$ \\
\hline Power (W) & $4.6 \mathrm{~W}$ & $4.6 \mathrm{~W}$ \\
\hline $\begin{array}{c}\text { Efficacy } \\
\text { (Lumens/watt) }\end{array}$ & $102.77 \mathrm{~lm} / \mathrm{W}$ & $102.77 \mathrm{~lm} / \mathrm{W}$ \\
\hline $\begin{array}{c}\text { LED luminaire } \\
\text { temperature } \\
\left({ }^{\circ} \mathrm{C}\right)\end{array}$ & $19.5^{\circ} \mathrm{C}$ & $14.8^{\circ} \mathrm{C}$ \\
\hline
\end{tabular}

In this paper the investigation methodologies are developed to advance both the experimental and theoretical descriptions of the LED luminaires. Through innovative carful experimental investigations, the commercial luminaires could be fully characterized. Becoming aware with the key parameters of the luminaires, an enhanced luminaire could be devised and implemented. Both the theoretical and the experimental results show that the developed luminaire has better performance parameters than standard commercial ones. If the LEDs become more efficient radiators it could be built a better lamp concerning the efficacy [12]. So, the road of the improvement is endless.

\section{REFERENCES}

[1] " Lighting Efficiency "2013" Dr Mohamed Helal", 14 Sep 2013,AEEE, pp.12,( https://www.scribd.com/doc/168176807/LightingEfficiency-2013-Dr-Mohamed-Helal\#download)

[2] ''LIGHTING OFFICES WITH LEDS: A STUDY ON RETROFITTING SOLUTIONS",2013, SPYROS STRAVORAVDIS, pp.227,(pdf)

[3] "Building Technologies Program, SOLID-STATE LIGHTING TECHNOLOGY FACT SHEET", January 2012, U.S Department of Energy, Energy Efficiency and Renewable Energy, pp.4, (pdf)

[4] "LED LUMINAIRE LIFETIME: Recommendations for Testing and Reporting”, June 2011, Next Generation Lighting Industry Alliance with the U. S. Department of Energy, pp. 30,(pdf).

[5] 'Luminaires", May 1997, Pacific Gas and Electric Company, pp. 7,(pdf), (http://www.pge.com/pec)

[6] "Simulating the illuminance and the efficiency of the LED and fluorescent lights used in indoor lighting design" , 10 November 2008, Chih-Hsuan Tsuei, JuiWen Pen, Wen-Shing Sun,pp.10,(pdf).

[7] "Illumination With Solid State Lighting Technology", MARCH/APRIL 2002, IEEE JOURNAL ON 
SELECTED TOPICS IN QUANTUM ELECTRONICS, Daniel A. Steigerwald, Jerome C. Bhat, Dave Collins, Robert M. Fletcher, Mari Ochiai Holcomb, Michael J. Ludowise, Member, IEEE, Paul S. Martin, and Serge L. Rudaz, pp.11,(pdf).

[8] "A COMPREHENSIVE STUDY OF AN OPTICAL TRANSCEIVER", April 1992, Abd El Halim Zekry and Abd Alrahman El-Amoud, pp.30.

[9] "A Simple Method for Comparative Study on the Thermal Performance of LEDs and Fluorescent Lamps",
2009, Yaxiao Qin, Student Member, IEEE, Deyan Lin, Member, IEEE, and S. Y. (Ron) Hui, Fellow, IEEE,pp.8

[10] “WinSpice3 User's Manual”,15 March, 2001,Mike Smith,pp.139,(pdf)

[11] "LED_Luminaire_Design_Guide",2007-2012, Cree, pp.18,(pdf)

[12] "Sustainability, Energy and Architecture, Case Studies in Realizing Green Buildings", 2014, Elsevier, Ali Sayigh, pp.527. 